\title{
Hot Deformation Behavior and Constitutive Modelling of Low Carbon Micro-alloyed Steel YQ450NQR1 During Isothermal Compression
}

\author{
Kun Dou ${ }^{1 *}$, Qing Liu ${ }^{2}$ \\ Corresponding Author: Dr. Kun Dou. \\ E-mail: Kun.Dou@brunel.ac.uk \\ Tel: $+44(0) 1895267054$
}

1. Brunel Center for Advanced Solidification Technology (BCAST), Brunel University London, Uxbridge, Middlesex, London, UB8 3PH, UK.

2. State Key Laboratory of Advanced Metallurgy, University of Science and Technology Beijing, Beijing, 100083, China.

\begin{abstract}
In this paper, the flow stress curves of YQ450NQR1 low carbon (wt. 0.12\%) vanadium micro-alloyed YQ450NQR1 steel are obtained by conducting isothermal compression tests using Gleeble-1500 thermal simulation machine covering the temperature range of $1143 \mathrm{~K}-1433 \mathrm{~K}$. To better describe the complex and abrupt deformation during the hot rolling process of the "Z-shaped" YQ450NQR1 products used for train beams, three deformation strain rates $\left(1 \mathrm{~s}^{-1}, 10 \mathrm{~s}^{-1}\right.$ and $\left.30 \mathrm{~s}^{-1}\right)$ are selected to study the hot deformation behavior. On this basis, a constitutive model considering deformation activation energy is established from the experimental stress-strain data. The model is further validated against multiple sets of hot compression test data and shows a good accuracy between predicted and tested flow stress with a correlation coefficient of 0.988 and an average error of $8.4 \%$, which indicates the accuracy and reliability of the constitutive model for YQ450NQR1 steel.
\end{abstract}

Key Words: Steel; Flow stress; Constitutive equation; YQ450NQR1; Hot deformation 


\section{Introduction}

YQ450NQR1 is a type of low carbon micro-alloyed steel, which is widely used in production of train beams. Microstructures and high temperature mechanical properties as well as the fracture mechanism concerning this type steel have been widely studied. For example, Dou et al. ${ }^{[1]}$ have studied the micro-segregation behavior of solute elements and internal crack susceptibility of this steel in the continuous casting process, on this basis, the secondary phase precipitation behavior under various cooling rate in steel solidification process are analyzed ${ }^{[2-4]}$. However, few researchers have paid attention to the hot deformation behavior of low carbon micro-alloyed steel in casting and rolling process. For curved continuous casting machine, high temperature deformation occurs at the bending/straightening position after secondary cooling. It is widely accepted that improper deformation during casting would lead to serious billet surface cracks ${ }^{[5-9]}$. Moreover, for the hot rolling process of YQ450NQR1 steel, the bloom with a cross-section size of $360 \mathrm{~mm} \times 450 \mathrm{~mm}$ is rolled into the "Z-shape" beam with a thickness of $10 \mathrm{~mm}$ ${ }^{[10]}$, two of piece of them are later welded together to form the final train beam (Fig.1). Since the deformation process of YQ450NQR1 steel from continuous casting bloom to hot rolled beam is complex, it is necessary to include a wide range of deformation parameters for research.

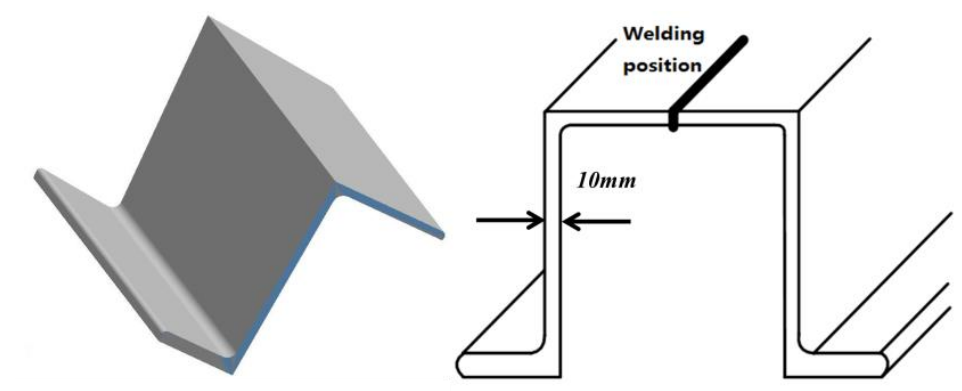

Figure. 1. Shape and size of "Z-shape" beam

Considering above facts, the main objective of this paper is to study the hot deformation behavior of YQ450NQR1 steel and establishing a reliable constitutive model to evaluate and predict the effect of process parameters on hightemperature flow behavior in casting/rolling process. To achieve this goal, isothermal hot compression tests are conducted over a wide range of strain-rates and temperatures. The experimental data are then used to formulate a mathematical relationship concerning temperature, strain rate and stress. Based on that, the constitutive model is validated against experimental results.

\section{Experiments}

The sample for hot compression test $(\phi 8 \mathrm{~mm} \times 15 \mathrm{~mm})$ is machined from YQ450NQR1 steel. Table 1 shows its chemical compositions. Gleeble 1500 thermal simulation machine is used for experiment. 
Table 1 Chemical compositions of YQ450NQR1 steel (wt \%)

\begin{tabular}{ccccccccc}
\hline Steel & $\mathrm{C}$ & $\mathrm{Si}$ & $\mathrm{Mn}$ & $\mathrm{P}$ & $\mathrm{S}$ & $\mathrm{V}$ & $\mathrm{N}$ & $\mathrm{Fe}$ \\
\hline YQ450NQR1 & 0.12 & 0.45 & 1.26 & 0.02 & 0.01 & 0.12 & 0.013 & Bal. \\
\hline
\end{tabular}

As is shown in Fig. 2, the sample is first pre-heated to $1373 \mathrm{~K}$ at $20 \mathrm{~K} / \mathrm{s}$ and then heated to $1573 \mathrm{~K}$ at $10 \mathrm{~K} / \mathrm{s}$, after which the sample temperature is kept constant for 180 seconds to guarantee solute elements distribution are homogenized. The sample is then cooled to the deformation temperature with a cooling rate of $3 \mathrm{~K} / \mathrm{s}$. The tests are performed under temperatures of $1143 \mathrm{~K}, 1173 \mathrm{~K}, 1203 \mathrm{~K}, 1233 \mathrm{~K}, 1293 \mathrm{~K}, 1338 \mathrm{~K}, 1383 \mathrm{~K}$ and $1433 \mathrm{~K}$. The tested strain rates are $1 \mathrm{~s}^{-1}, 10 \mathrm{~s}^{-1}, 30 \mathrm{~s}^{-1}$ and the total deformation amount is $60 \%$.

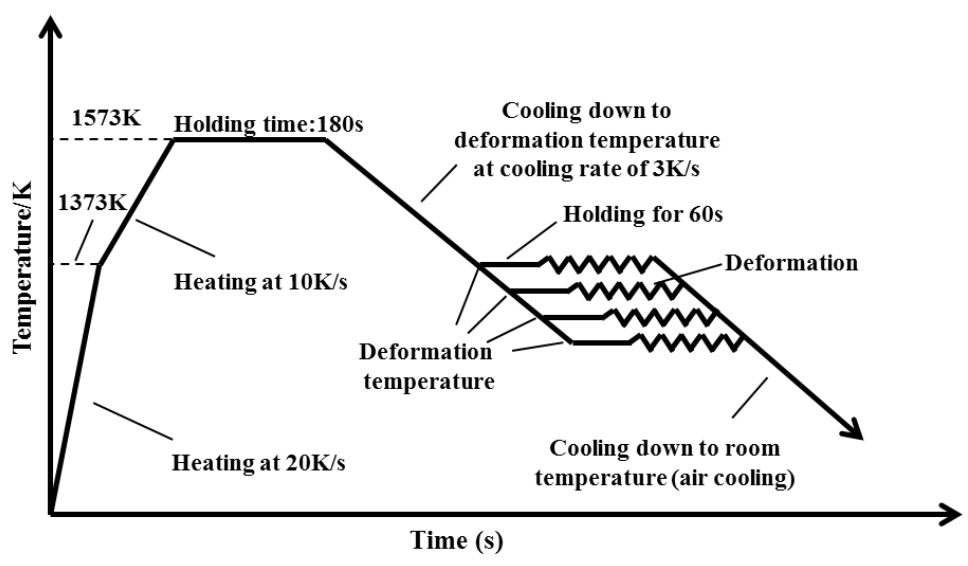

Figure.2 Schematic diagram of the experimental heat cycle

\section{Results}

Various flow stress curves of YQ450NQR1 micro-alloyed steel at different strain rates are shown in Fig. 3 (a)-(c). When strain rate is fixed, the stress first increases with strain and then reach a steady state, which is a common sign of elastic-plastic deformation. As the test temperature increases, i.e. 1433K, the stress in the final deformation stage would undergo a decrease owing to the softening of materials at elevated temperatures. With the increase in strain rate, the stress at certain temperature rises and the high temperature softening behavior disappears.
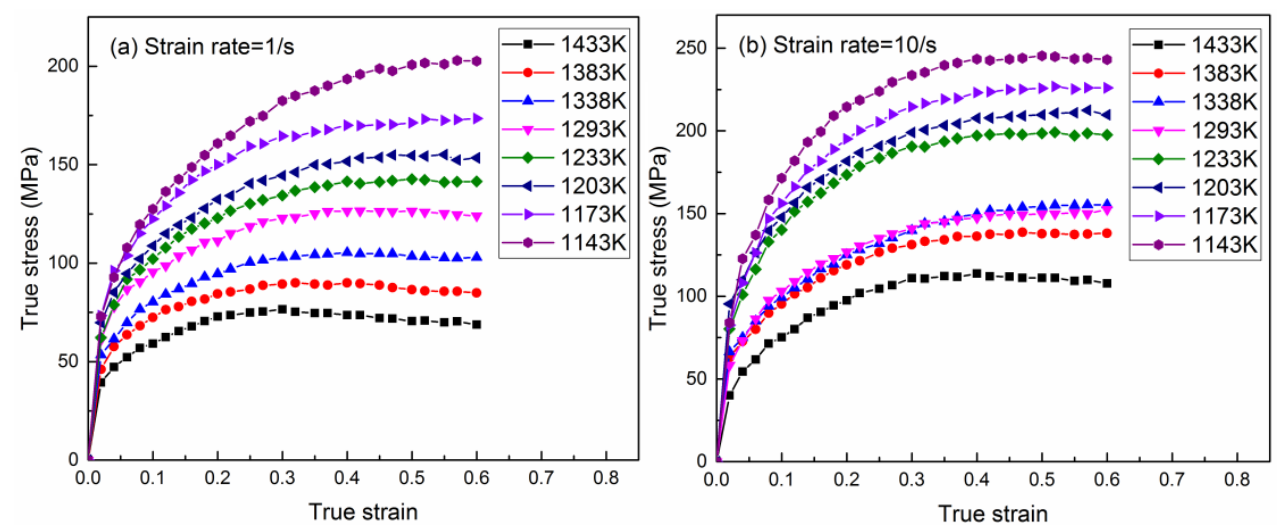


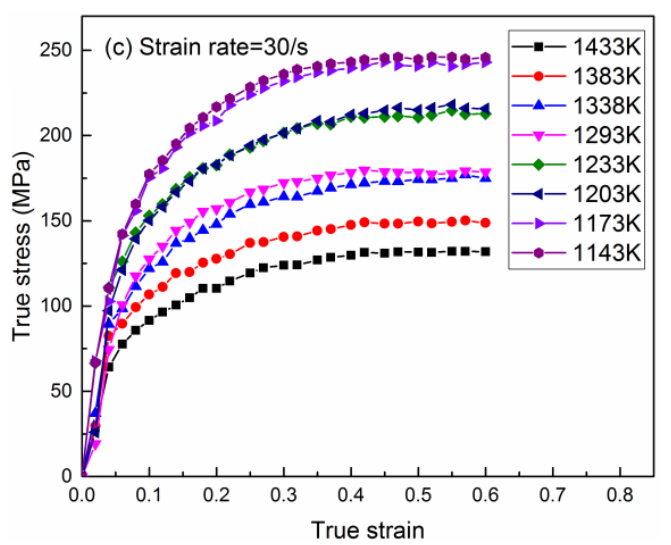

Figure.3 The stress-strain curves of YQ450NQR1 steel bloom at various strain rates

According to relative research proposed by other researchers ${ }^{[1-13]}$, the typical flow stress for metals would experience three stages, which is work hardening, dynamic recovery and dynamic recrystallization. With variable strain rate and test temperature, the specific curve shapes would change accordingly. The nature in that is because lower strain rate would offer longer time for the occurrence of dynamic recovery and dynamic recrystallization. While higher deformation temperatures bring more energy regarding grain boundary slip/dislocation as well as voids motion ${ }^{[14-17]}$.

\section{Discussion}

\subsection{Constitutive analysis}

\subsubsection{Establishment of constitutive equation}

Hot deformation of metals is usually activated by thermal phenomenon, which could be described via relevant thermodynamic parameters. In the process of metal thermal deformation, flow stress $\sigma$ is mainly dependent on strain rate $\dot{\varepsilon}$ and deformation temperature $T$. The effects of strain rate $\dot{\varepsilon}$ and deformation temperature $T$ on flow stress $\sigma$ could typically be described by the following kinetic equations ${ }^{[18]}$.

At lower deformation state $(\alpha \sigma<0.8)$, the relation between flow stress and strain rate could be described in form of Eq. (1).

$\dot{\varepsilon}=A_{1} \sigma^{n_{1}}$

At higher deformation state $(\alpha \sigma>1.8)$, the relation between flow stress and strain rate could be described in form of Eq. (2).

$\dot{\varepsilon}=A_{2} \exp (\beta \sigma)$

In a later study, some researchers ${ }^{[19-21]}$ considered the influence of thermal deformation activation energy $Q$ as well as temperature $T$. And the relation between flow stress and strain rate could be described in form of Eq. (3). $\dot{\varepsilon}=A[\sinh (\alpha \sigma)]^{n} \exp (-Q / R T)$ 
As is proved by many experimental results ${ }^{[22-25]}$, Eq. (3) could describe the relation between flow stress and strain rate efficiently at both low stress and high stress conditions.

In Eq. (1)-(3), $A_{1}, A_{2}, \mathrm{~A}, \mathrm{n} 1, \mathrm{n}, \alpha, \beta$ are material constants which is not dependent on test temperatures but material properties, among which the values of $\alpha, \beta$ and $n 1$ are related by Eq. (4) as follows ${ }^{[21]}$.

$\alpha=\frac{\beta}{n_{1}}$

$Q$ is activation energy for hot deformation process, which mainly describes the correlation between work hardening and dynamic softening, $\mathrm{KJ} / \mathrm{mol}$.

$T$ is deformation temperature, $\mathrm{K}$.

$R$ is universal gas constant with a value of $8.314 \mathrm{~J} /(\mathrm{mol} \cdot \mathrm{K})$,

In 1944, Zener and Hollomon ${ }^{[22]}$ conducted a series of isothermal deformation tests for metals, after which they have been able to verify that strain rate and deformation temperature determines the interrelation between stress and strain. They described it as Zener-Hollomon coefficient, as is shown as Eq. (5)

$Z=\dot{\varepsilon} \exp (Q / R T)$

Combining Eq. 4 and Eq.5, it could be noticed that once the values for $A, n, \alpha$ and $Q$ are settled, the flow stress under any condition could be solved accordingly, which is a crucial factor for the designing and optimization of materials processing technology.

In order to determine for materials constant for YQ45ONQR1 steel in this study, the $\beta$ and $n_{1}$ values should be known first, then $\alpha$ value could be calculated by Eq. 4 .

Assuming the inter-relation curves between $\ln [\sinh (\alpha \sigma)]$ and $\ln \dot{\varepsilon}$ are reasonably close to linear and parallel to each other, the $\alpha$ value could be calculated accordingly ${ }^{[23]}$.

By assuming that activation energy $Q$ is not dependent on deformation temperature $T$ in the calculation region, the following Eq. (6)-(8) could be obtained by taking natural logarithm of Eq. (1), Eq. (2) and Eq.(3), respectively.

$$
\begin{aligned}
& \ln \dot{\varepsilon}=\ln A_{1}+n_{1} \ln \sigma \\
& \ln \dot{\varepsilon}=\ln A_{2}+\beta \sigma \\
& \ln \dot{\varepsilon}=\ln A-\frac{Q}{R T}+n \ln [\sinh (\alpha \sigma)]
\end{aligned}
$$

Using the hot deformation tests curves from Fig. 3 and implementing the flow stress and strain data into Eq. (6)(8). The relationship between $\ln \dot{\varepsilon}$ and $\ln \sigma$ is obtained as Fig. 4. In the next step, the $\ln \dot{\varepsilon} \sim \sigma$ curves are calculated as Fig. 5. Obviously both data set show a linear relationship, and the slopes of Eq. (6) and Eq. (7) are the values 
of $n_{l}$ and $\beta$. Taking linear regression for Eq. (6) and Eq. (7) and averaging the fitted slopes, we obtain that $n_{l}=$ 8.65592 and $\beta=0.06644$. Consequently, the value of $\alpha$ is determined by Eq. (4), which is 0.00768 .

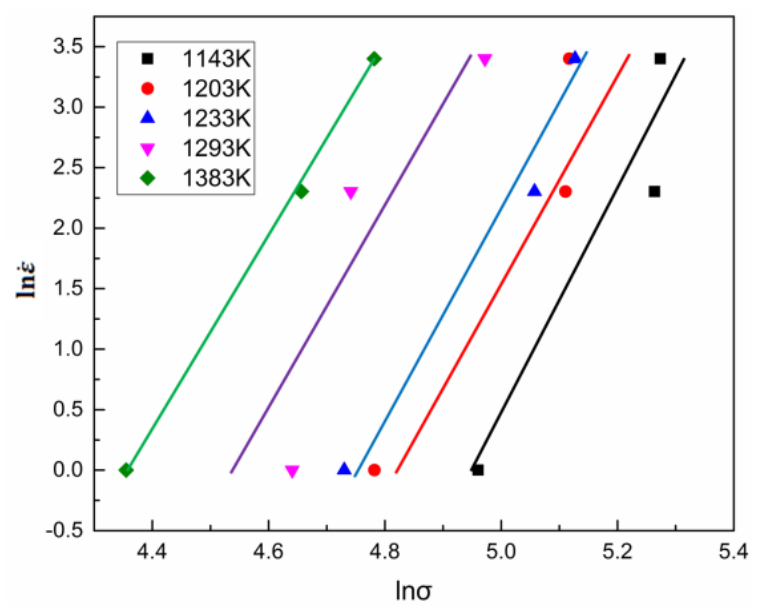

Figure. $4 \ln \dot{\varepsilon} \sim \ln \sigma$ relation curve

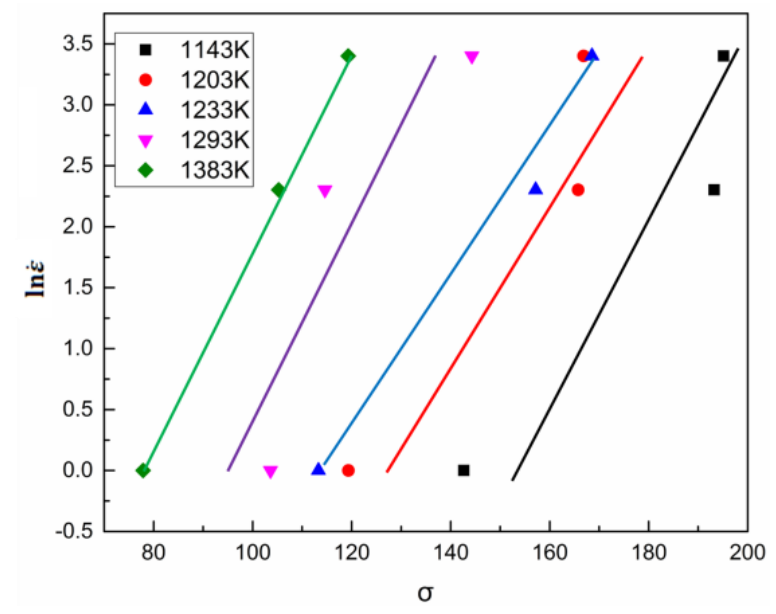

Figure. $5 \ln \dot{\varepsilon} \sim \sigma$ relation curve

On this basis, Eq. (9) is obtained by taking partial derivatives for Eq. (3).

$Q=R\left\{\frac{\partial \ln \dot{\varepsilon}}{\partial \ln [\sinh (\alpha \sigma)]}\right\}_{T}\left\{\frac{\partial \ln [\sinh (\alpha \sigma)]}{\partial(1000 / T)}\right\}_{\dot{\varepsilon}}$

Here we define $Q_{1}=\left\{\frac{\partial \ln \dot{\varepsilon}}{\partial \ln [\sinh (\alpha \sigma)]}\right\}_{T}$ which is slope of the $\ln [\sinh (\alpha \sigma)] \sim \ln \dot{\varepsilon} \operatorname{linear}$ curve at fixed deformation temperature. Then we define $Q_{2}=\left\{\frac{\partial \ln [\sinh (\alpha \sigma)]}{\partial(1000 / T)}\right\}_{\dot{\varepsilon}}$ which is the slope of $1000 / T \sim \ln [\sinh (\alpha \sigma)]$ linear curve under fixed strain rate. $\ln \mathrm{A}-\frac{Q}{R T}$ is the intercept of the $\ln [\sinh (\alpha \sigma)] \sim \ln \dot{\varepsilon}$ linear curve considering Eq. (8).

Applying the experimental data of YQ450NQR1 hot deformation tests and combining Eq.(4), Eq. (8) and Eq. (9), the $\ln [\sinh (\alpha \sigma)] \sim \ln \dot{\varepsilon}$ and $1000 / T \sim \ln [\sinh (\alpha \sigma)]$ linear curve sets are obtained as Fig. 6 and Fig. 7. 


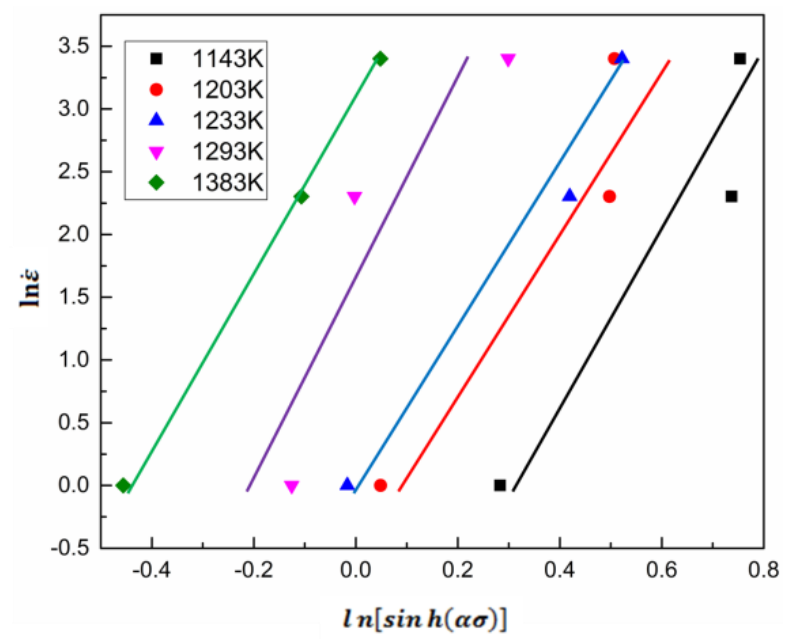

Figure. $6 \ln [\sinh (\alpha \sigma)] \sim \ln \dot{\varepsilon}$ relation curve

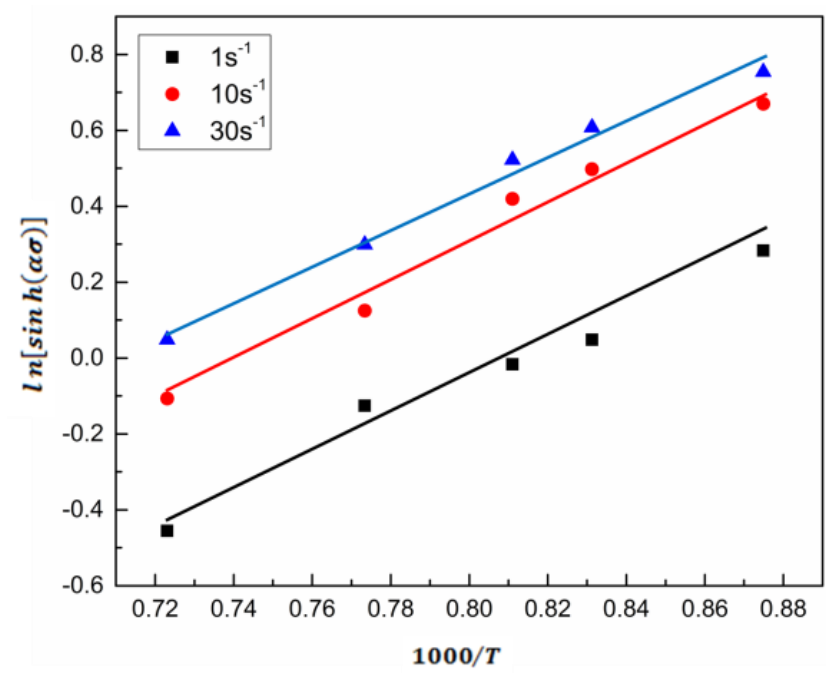

Figure.7 1000/T $\sim \ln [\sinh (\alpha \sigma)]$ relation curve

By applying linear regression, averaging slopes of Fig. 6 and Fig. 7, we obtain $n=Q_{I}=\left\{\frac{\partial \ln \dot{\varepsilon}}{\partial \ln [\sinh (\alpha \sigma)]}\right\}_{T}=6.49330$, $Q_{2}=\left\{\frac{\partial \ln [\sinh (\alpha \sigma)]}{\partial(1000 / T)}\right\}_{\dot{\varepsilon}}=5.05561, Q=272.93 \mathrm{KJ} \mathrm{mol}^{-1}$. According to Eq. (8), the average value for $\ln A-\frac{Q}{T}$ is 3.83. Furthermore, $\ln A$ values under different temperatures are determined on the basis of $Q, R$ and $T$ values. The mean value for $\ln A$ is 22.52 hence the value of $A$ is $6.03 \times 10^{9}$.

Solution for values of $\alpha, n, Q$ and $\ln A$ are repeated using data under various strain condition $(\varepsilon=0.14,0.2,0.27$, $0.35,0.42,0.5,0.57)$. The relationships between the calculated materials constant values and true strains are shown in Fig. 8. A fifth order polynomial fitting curve is found to be consistent with the influence of strain on material constants showing good correlations. The results display agreement with previous related research such as those from Li et al. ${ }^{[24]}$ and Silva et al. ${ }^{[25]}$. 

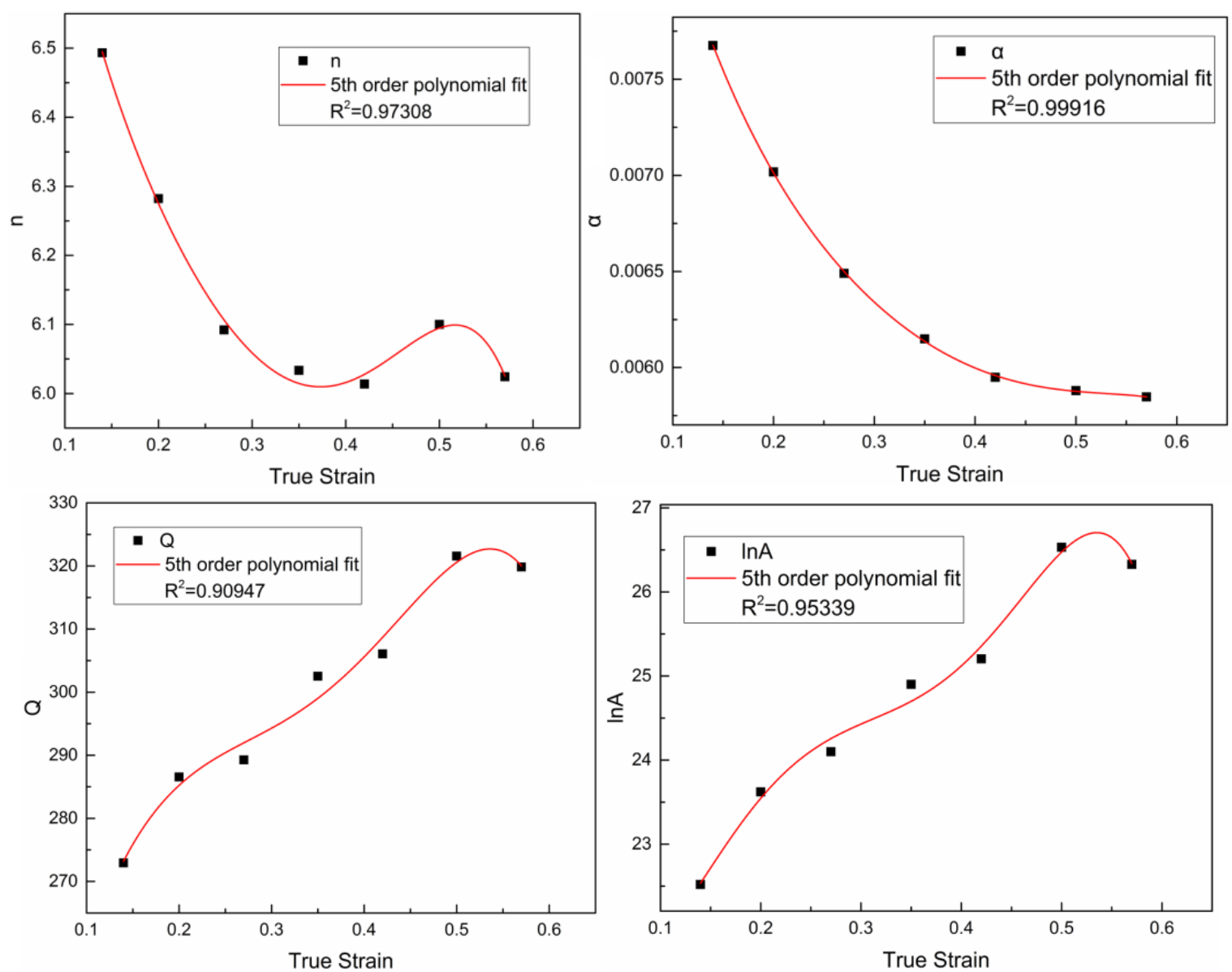

Figure. 8 The relationship between the $\mathrm{n}, \alpha$, Q and $\ln \mathrm{A}$ values vs true strains

And the final polynominal relationships regarding $n, \alpha, Q, \ln A$ and strain are shown as Eq. (10)-(13), respectively.

The fitting coefficients in above equations are listed in Table 2.

$$
\begin{aligned}
& n=n_{1}+n_{2} \varepsilon+n_{3} \varepsilon^{2}+n_{4} \varepsilon^{3}+n_{5} \varepsilon^{4}+n_{6} \varepsilon^{5} \\
& \alpha=\alpha_{1}+\alpha_{2} \varepsilon+\alpha_{3} \varepsilon^{2}+\alpha_{4} \varepsilon^{3}+\alpha_{5} \varepsilon^{4}+\alpha_{6} \varepsilon^{5} \\
& Q=Q_{1}+Q_{2} \varepsilon+Q_{3} \varepsilon^{2}+Q_{4} \varepsilon^{3}+Q_{5} \varepsilon^{4}+Q_{6} \varepsilon^{5} \\
& \ln A=A_{1}+A_{2} \varepsilon+A_{3} \varepsilon^{2}+A_{4} \varepsilon^{3}+A_{5} \varepsilon^{4}+A_{6} \varepsilon^{5}
\end{aligned}
$$

Table 2 Coefficients of the polynomial functions

\begin{tabular}{cccc}
\hline$\alpha$ & $A$ & $n$ & $Q$ \\
\hline$\alpha_{1}=0.0109$ & $A_{1}=23.593$ & $n_{1}=7.7362$ & $Q_{1}=189.54$ \\
$\alpha_{2}=-0.0358$ & $A_{2}=68.302$ & $n_{2}=-16.153$ & $Q_{2}=993.72$ \\
$\alpha_{3}=0.1254$ & $A_{3}=776.07$ & $n_{3}=79.725$ & $Q_{3}=-3326.7$ \\
$\alpha_{4}=-0.2761$ & $A_{4}=-3164.4$ & $n_{4}=-253.25$ & $Q_{4}=2845.6$ \\
$\alpha_{5}=0.3495$ & $A_{5}=5668.9$ & $n_{5}=437.92$ & $Q_{5}=6373.5$ \\
$\alpha_{6}=-0.1849$ & $A_{6}=-3703.8$ & $n_{6}=-294.74$ & $Q_{6}=-9221.6$ \\
\hline
\end{tabular}


Combining Eq. (3) and (5), we obtain Eq. (14),

$\left(\frac{Z}{A}\right)^{\frac{1}{n}}=\sinh (\alpha \sigma)$

Hence, flow stress could be expressed in Zenner-Hollomon form (Eq. (15)) based on Eq. (3), Eq. (5) and Eq. (14).

$\sigma=\frac{1}{\alpha} \ln \left\{\left(\frac{Z}{A}\right)^{\frac{1}{n}}+\left[\left(\frac{Z}{A}\right)^{\frac{2}{n}}+1\right]^{\frac{1}{2}}\right\}=\frac{1}{\alpha} \ln \left\{\left(\frac{\dot{\varepsilon} \exp (Q / R T)}{A}\right)^{\frac{1}{n}}+\left[\left(\frac{\dot{\varepsilon} \exp (Q / R T)}{A}\right)^{\frac{2}{n}}+1\right]^{\frac{1}{2}}\right\}$

Within the experimental strain $\varepsilon$ range of $0-0.6$, the stress value could be determined at certain condition with Eq.

\subsubsection{Validation of constitutive model}

In order to validate the model prediction precision, 200 groups of tensile tests with 7 levels of strain $(\varepsilon=0.15$, $0.20,0.25,0.35,0.40,0.50,0.58)$ are carried out and the experimental stress-strain relationships are obtained. In the meantime, the tensile tests are modelled using the constitutive model established above. Finally, the stressstrian relationships between experiments and modelling are further compared. Fig. 9 shows the correspondence between prediction and experiments regarding flow stress.
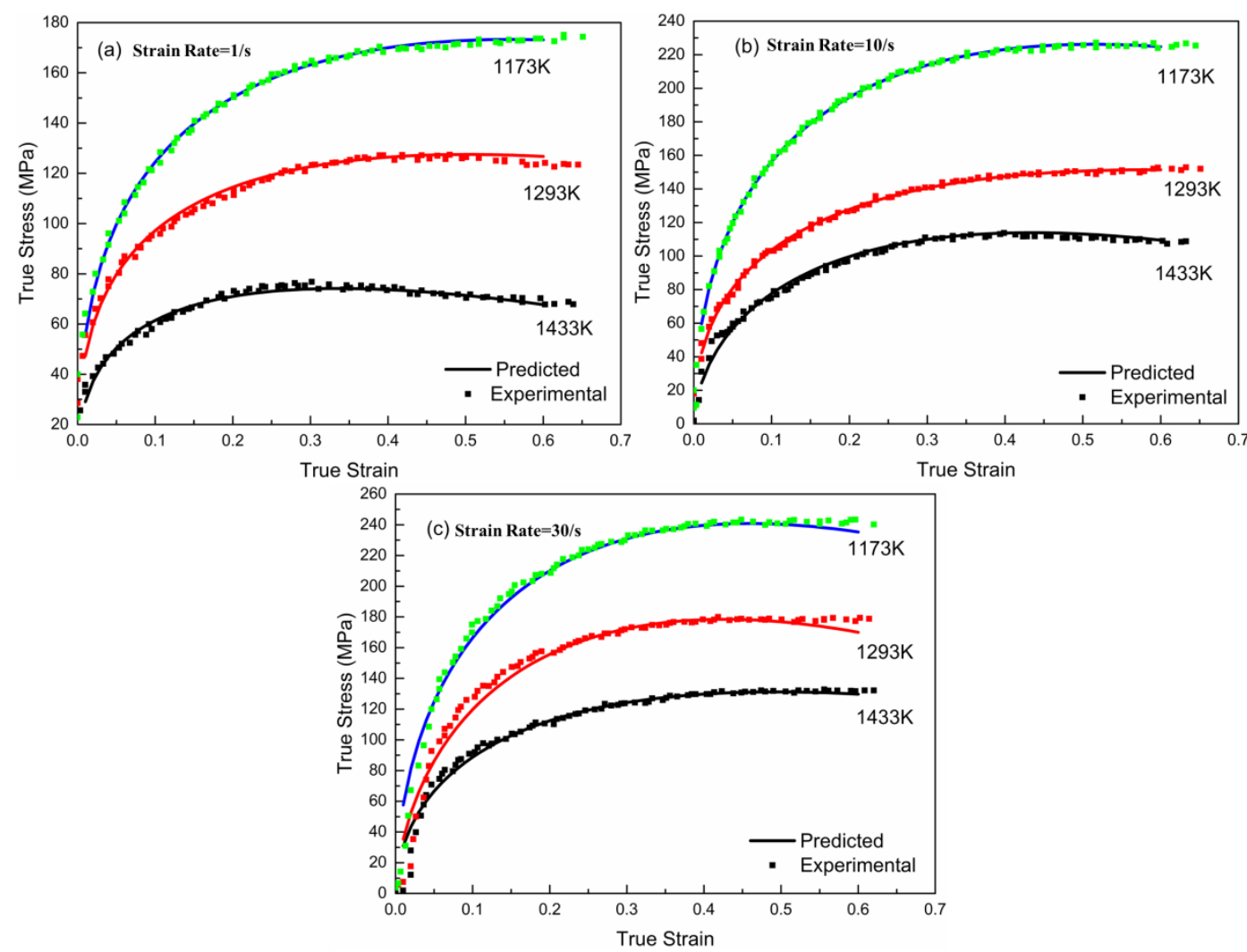

Figure. 9 Comparisons of predicted values and actual values under different deformation conditions 
As is seen in Fig. 9, the constitutive equation for YQ450NQR1 steel shows good prediction accuracy under most of the test conditions. However, Some variation occurs at work hardening regions in flow curves, which has also been reported by relevant researchers such as Cai et al. ${ }^{[26]}$ and Lin et al. ${ }^{[27]}$. The reason for this variation owns to the fact that Eq. (1) is only applicable to low stress conditions while $n_{l}$ value under high stress conditions are obtained based on that, which furthers affects the values of $\alpha$ and $Q$ as well as the accuracy of Eq. (15).

Furthermore, the stability of the constitutive equation for YQ450NQR1 steel is tested using correlation coefficient (R, Eq. (16)) and average absolute relative error (AARE, Eq. (17)).

$\mathrm{R}=\frac{\sum_{i=1}^{i=N}\left(\sigma_{\text {exp }}^{i}-\bar{\sigma}_{\text {exp }}\right)\left(\sigma_{p}^{i}-\bar{\sigma}_{p}\right)}{\sqrt{\sum_{i=1}^{i=N}\left(\sigma_{\text {exp }}^{i}-\bar{\sigma}_{\text {exp }}\right)^{2} \sum_{i=1}^{i=N}\left(\sigma_{p}^{i}-\bar{\sigma}_{p}\right)^{2}}}$

$\mathrm{AARE}=\frac{1}{N} \sum_{i=1}^{i=N}\left|\frac{\sigma_{\text {exp }}^{i}-\sigma_{p}^{i}}{\sigma_{\text {exp }}^{i}}\right| \times 100 \%$

Fig. 10 shows the correspondence between prediction and experiments. The calculated $\mathrm{R}$ value is 0.988 and AARE value is $8.40 \%$, meaning the established constitutive equation for YQ450NQR1 steel is reliable in flow stress prediction in the strain range of 0-0.6.

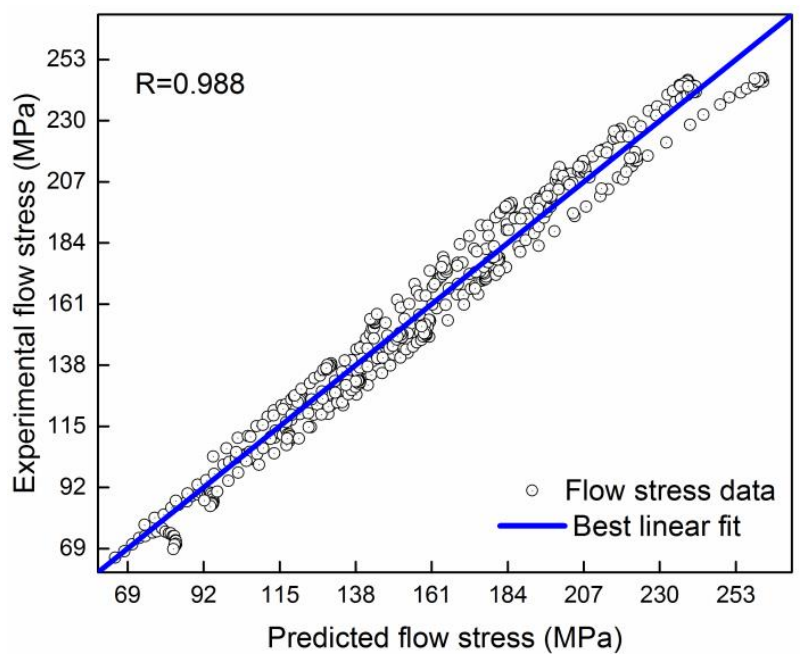

Figure. 10 Comparison between predicted and experimental data

\section{Conclusions}

In this work, the hot deformation behavior of low carbon vanadium micro-alloyed YQ450NQR1 steel is researched by performing thermal tests under the condition of temperatures range $1143 \mathrm{~K}-1433 \mathrm{~K}$, strain rate of $1 \mathrm{~s}^{-1}, 10 \mathrm{~s}^{-1}, 30 \mathrm{~s}^{-}$ ${ }^{1}$ and maximum strain of 0.6. On this basis, a constitutive equation for flow stress prediction is established combining experimental data and mathematical solution. The correlation coefficient and the average absolute relative error are $0.988 \%$ and $8.40 \%$, respectively. Thus, the established constitutive model shows good correspondence with experimental data and proves to be helpful in the designing of hot rolling process for YQ450NQR1 steel. The research work carried out in this paper offers the theoratical foundation for study of 
thermal plastic deformation behavior for the low carbon vanadium micro-alloyed with complex shape and significacnt deformation. In the future work, the established model will be further addressed and applied in the modelling of steel rolling process using finite element method, based on which, the optimization of rolling process parameters (rolling temperatures, deformation extent, strain rate etc.) will be discussed and carried out.

\section{Acknowledgement}

The authors would like to acknowledge the financial support provided by Key Research \& Development Foundation from State Key Laboratory of Advanced Metallurgy, University of Science and Technology Beijing, China [41614014] and Independent Research \& Development Foundation from State Key Laboratory of Advanced Metallurgy, University of Science and Technology Beijing, China [41602023].

\section{Conflict of Interest:}

The authors declare that they have no conflict of interest. 


\section{References}

[1] K. Dou, J. Qing, L. Wang, X. Zhang, B. Wang, Q. Liu, H. Dong, Acta Metall. Sin. 2014, 50,12.

[2] K. Dou, L. Meng, Q. Liu, B. Liu, Y. Huang, Met. Mater. Int., 2016, 22, 3.

[3] K. Dou, Z. Yang, Q. Liu, Y. Huang, H. Dong, High Temp. Mat. Pr., 2017, 36, 7.

[4] K. Dou, L. Wang, J. Qing, X. Zhang, B. Wang, B. Liu, Q. Liu, in Advances in the Science and Engineering of Casting Solidification, Springer, Cham, 2015.

[5] M. L. Zappulla, B. G. Thomas, In TMS 2017 146th Annual Meeting \& Exhibition Supplemental Proceedings (pp. 501-510), Springer, Cham, 2017.

[6] B. Mintz, S. Yue, J. J. Jonas, Int. Mater. Rev., 1991, 36, 1.

[7] B. Mintz, J. M. Arrowsmith, Met. Technol., 1979, 6, 1.

[8] H. Kametani, Metall. Mater. Trans. B, 1998, 29, 6.

[9] J. Shen, M. Long, D. Chen, J. Zhang, Z. Dong, Eng. Fail. Anal., 2016, 66.

[10] J. Qing, , X. Duan, M. Xiao, J. Li, M. Liu, Q. Liu, H. Shen, Strength Mater+, 2018, 1.

[11] Z. Akbari, H. Mirzadeh, J. Cabrera, Mater. Design, 2015, 77.

[12] Z. Liang, Y. Li, M. Huang, Scripta Mater., 2016, 112.

[13] H. Mirzadeh, Metall. Mater. Trans. A, 2015, 46, 9.

[14] A. Laasraoui, J. Jonas, ISIJ Int., 1991, 31, 1.

[15] G. Dini, R. Ueji, A. Najafizadeh, S. M. Monir-Vaghefi, Mat. Sci. Eng. A-Struct., 2010, A527.

[16] E. Farabi, A. Zarei-Hanzaki, H. R. Abedi, Adv. Eng. Mater., 2016, 18, 4.

[17] A. K. Gupta, S. K. Singh, S. Reddy, G. Hariharan, Mater. Design, 2012, 35.

[18] Y. Lu, Z. Zhu, D. Li, Q. Xie, Mat. Sci. Eng. A-Struct., 2017, 679.

[19] H. Conrad, Acta Metall., 1963, 11, 1.

[20] X. L. Fang, D. J. Jiang, J. Mater. Sci., 2011, 46, 10.

[21] Y. Cao, H. S. Di, R. D. K. Misra, High Temp. Mat. Pr., 2015, 34, 2.

[22] C. Zener, J. H. Hollomon, J. Appl. Phys., 1944, 15, 1.

[23] D. Y. Ju, W. M. Zhang, Y. Zhang, Mater. Sci. Eng. A-Struct., 2006, 438.

[24] L. Li, J. Zhou, J. Duszczyk, J. Mater. Process. Technol., 2016, 172, 3.

[25] R. Da Silva Septimio, S. T. Button, C. J. Van Tyne, J. Mater. Sci., 2016, 51, 5.

[26] A. Hajari, M. Morakabati, S. M. Abbasi, H. Badri, Mat. Sci. Eng. A-Struct., 2017, 681.

[27] Y. Lin, M. Chen, J. Zhong, Comput. Mater. Sci., 2008, 42, 3. 


\section{Figures and Tables Captions}

Figure.1. Shape and size of "Z-shape" beam

Figure.2 Schematic diagram of the experimental heat cycle

Figure.3 The stress-strain curves of YQ450NQR1 steel bloom at various strain rates

Figure. $4 \ln \dot{\varepsilon} \sim \ln \sigma$ relation curve

Figure. $5 \ln \dot{\varepsilon} \sim \sigma$ relation curve

Figure. $6 \ln [\sinh (\alpha \sigma)] \sim \ln \dot{\varepsilon}$ relation curve

Figure.7 1000/T $\sim \ln [\sinh (\alpha \sigma)]$ relation curve

Figure. 8 The relationship between the $\mathrm{n}, \alpha, \mathrm{Q}$ and $\ln \mathrm{A}$ values vs true strains

Figure. 9 Comparisons of predicted values and actual values under different deformation conditions

Figure. 10 Comparison between predicted and experimental data

Table 1 Chemical compositions of YQ450NQR1 steel (wt \%)

Table 2 Coefficients of the polynomial functions 